\title{
ExoMol line lists - XXXVIII. High-temperature molecular line list of silicon dioxide $\left(\mathrm{SiO}_{2}\right)$
}

\author{
A. Owens, ${ }^{1 \star}$ E.K. Conway, ${ }^{1,2}$ J. Tennyson ${ }^{1} \dagger$ and S. N. Yurchenko ${ }^{1} \ddagger$ \\ ${ }^{1}$ Department of Physics and Astronomy, University College London, Gower Street, WC1E 6BT London, UK \\ 2 Atomic and Molecular Physics Division, Center for Astrophysics | Harvard \& Smithsonian, Cambridge, Massachusetts 02138, USA
}

Accepted XXXX. Received XXXX; in original form XXXX

\begin{abstract}
Silicon dioxide $\left(\mathrm{SiO}_{2}\right)$ is expected to occur in the atmospheres of hot rocky superEarth exoplanets but a lack of spectroscopic data is hampering its possible detection. Here, we present the first, comprehensive molecular line list for $\mathrm{SiO}_{2}$. The line list, named OYT3, covers the wavenumber range $0-6000 \mathrm{~cm}^{-1}$ (wavelengths $\lambda>1.67 \mu \mathrm{m}$ ) and is suitable for temperatures up to $T=3000 \mathrm{~K}$. Almost 33 billion transitions involving 5.69 million rotation-vibration states with rotational excitation up to $J=$ 255 have been computed using robust first-principles methodologies. The OYT3 line list is available from the ExoMol database at www.exomol.com.
\end{abstract}

Key words: molecular data - opacity - planets and satellites: atmospheres - stars: atmospheres - ISM: molecules.

\section{INTRODUCTION}

In the gas phase, silicon dioxide $\left({ }^{28} \mathrm{Si}^{16} \mathrm{O}_{2}\right)$ is a linear triatomic molecule, analogous to $\mathrm{CO}_{2} . \mathrm{SiO}_{2}$ is expected to be present in the atmospheres of hot rocky super-Earth exoplanets (Tennyson \& Yurchenko 2017a). These tidally-locked exoplanets are in close proximity to their host star with their dayside exposed to temperatures reaching $4000 \mathrm{~K}$. At such high temperatures the material on the surface of the planet vaporises to produce an atmosphere strongly dependent on initial planetary composition (Schaefer \& Fegley 2009; Miguel et al. 2011), e.g. composed of $\mathrm{SiO}_{2}$-rich silicates like the Earth's continental crust (Schaefer, Lodders \& Fegley 2012). Furthermore, the likely presence of water vapour creates a steam atmosphere, and since all major rock-forming elements ( $\mathrm{Si}, \mathrm{Mg}$, $\mathrm{Ca}$, etc.) dissolve in steam to some extent, one can expect to encounter simple molecules composed of rock-forming elements with oxygen and hydrogen (Fegley et al. 2016).

Investigating the spectroscopy of hot rocky super-Earths requires accurate molecular opacities on systems such as $\mathrm{SiO}_{2}$. There is, however, very limited information on some of these molecules, partly because they form in the gas phase at very high temperatures making their spectra challenging to measure in the laboratory. Instead, theory offers a more viable route for generating the molecular line lists of these systems through systematic approaches based on first-principles methodologies (Tennyson 2016; Tennyson \& Yurchenko 2017b). These computational procedures have been successfully adopted by the ExoMol database (Tennyson \& Yurchenko 2012; Tennyson et al. 2016), which provides comprehensive line lists suitable for modelling exoplanet atmospheres at elevated temperatures. Already a large number of important diatomic and polyatomic species have been treated within the ExoMol framework (Tennyson \& Yurchenko 2018), and efforts are now being focused on molecules relevant to hot rocky super-Earth atmospheres. This brings about its own unique set of challenges, notably the completeness of the line list at very high temperatures and the lack of experimental data to refine the theoretical spectroscopic model.

Regarding $\mathrm{SiO}_{2}$, only a few studies have investigated its infrared spectrum with measurements of the $\nu_{2}$ bending mode (Andrews \& McCluskey 1992) and $\nu_{3}$ stretching mode (Schnöckel 1978; Schnöckel 1980). However, since these studies were performed in solid argon matrices the measured wavenumbers can be shifted by tens of wavenumbers (Jacox 1994) making it

* The corresponding author: alec.owens.13@ucl.ac.uk

$\dagger$ The corresponding author: j.tennyson@ucl.ac.uk

$\ddagger$ The corresponding author: s.yurchenko@ucl.ac.uk

(C) 2020 The Authors 
difficult to assess the usefulness of the determined values for gas phase studies. Similarly, only a small number of theoretical studies have considered silicon dioxide (Kaufman, Muenter \& Klemperer 1967; Pacansky \& Hermann 1978; Wang et al. 1996; Brinkmann, Tschumper \& Schaefer III 1999; Kostko, Ahmed \& Metz 2009; Hao, Xie \& Schaefer III 2014), but none of these are particularly relevant to the work presented here, namely the high-accuracy calculation of its rotation-vibration spectrum.

In this work, we present the first, comprehensive rotation-vibration line list of gas-phase $\mathrm{SiO}_{2}$. The new line list has been computed using robust first-principles methodologies (Tennyson 2016) within the ExoMol computational framework (Tennyson \& Yurchenko 2017b) and adds to the other available silicon-bearing molecules in the ExoMol database: $\mathrm{SiH}_{4}\left(\mathrm{Owens}_{\mathrm{s}}\right.$ et al. 2017), SiH (Gorman, Yurchenko \& Tennyson 2019), SiO (Barton, Yurchenko \& Tennyson 2013), SiS (Upadhyay et al. 2018) and $\mathrm{SiH}_{2}$ (Clark et al. 2020).

\section{METHODS}

The computational approach used to produce the $\mathrm{SiO}_{2}$ line list is described in detail in the supplementary material and only a brief summary is provided here. Initially, high-level $a b$ initio methods were used to compute the potential energy surface (PES) and dipole moment surface (DMS) of the electronic ground state of $\mathrm{SiO}_{2}$. The PES was generated using explicitly correlated coupled cluster ( $\mathrm{CCSD}(\mathrm{T})-\mathrm{F} 12 \mathrm{~b})$ calculations with extrapolation to the complete basis set limit, and included several additive energy corrections to account for small effects like scalar relativity. This approach is capable of producing accurate PESs for closed-shell molecules that can reproduce fundamental term values to within $\pm 1 \mathrm{~cm}^{-1}$ on average (e.g. see Owens et al. (2018) and references within). Due to the lack of reliable experimental data for this molecule, the PES was not empirically refined. The DMS was computed using CCSD(T)-F12b with a large augmented correlation consistent basis set. It is now well established that transition intensities computed using ab initio DMSs are comparable to, and occasionally more reliable, than experiment (Yurchenko 2014; Tennyson 2014). Both surfaces were computed on the same grid of 15365 nuclear geometries and then fitted with suitable analytic representations for use in the next stage of the calculation process. The potential energy and dipole moment surfaces are provided as supplementary material along with Fortran routines to construct them.

Line list calculations employed the variational nuclear motion program TROVE (Yurchenko, Thiel \& Jensen 2007), which was extended to treat linear triatomic molecules in this work. Benchmarking was performed against the triatomic nuclear motion code DVR3D (Tennyson et al. 2004) to ensure the validity of the TROVE implementation. The ability to utilise two nuclear motion codes based on different methodologies proved highly beneficial and meant the theoretical spectroscopic model of $\mathrm{SiO}_{2}$ could be checked for consistency. This was particularly important given the lack of experimental data to compare against. A large symmetry-adapted basis set was used in the rovibrational calculations with convergence testing performed at different $J$ values.

The line list was computed with a lower state energy threshold of $h c \cdot 15000 \mathrm{~cm}^{-1}$ ( $h$ is the Planck constant and $c$ is the speed of light) and considered transitions up to $J=255$ in the $0-6000 \mathrm{~cm}^{-1}$ range. The energy levels and wavefunctions of $\mathrm{SiO}_{2}$ can be classified under the $\boldsymbol{C}_{2 \mathrm{v}}(\mathrm{M})$ molecular symmetry group (Bunker \& Jensen 1998). The nuclear spin statistical weights are $g_{\mathrm{ns}}=\{1,1,0,0\}$ for states of symmetry $\left\{A_{1}, A_{2}, B_{1}, B_{2}\right\}$, respectively. Thus, $B_{1}$ and $B_{2}$ states need not be computed and transitions follow the symmetry selection rules $A_{1} \leftrightarrow A_{2}$; and the standard rotational selection rules, $J^{\prime}-J^{\prime \prime}=$ $0, \pm 1, J^{\prime}+J^{\prime \prime} \neq 0$; where ' and " denote the upper and lower state, respectively. These representations are correlated to the $\boldsymbol{D}_{\infty \mathrm{h}}(\mathrm{M})$ irreducible representation, commonly used for linear molecules, as $A_{1} \leftrightarrow \Sigma_{g}^{+}$and $A_{1} \leftrightarrow \Sigma_{u}^{-}$. Another standard spectroscopic descriptor is the Kronig parity e/f (Brown et al. 1975), related to the total parity $+1\left(A_{1}\right)$ and $-1\left(A_{2}\right)$ as follows: the parity of the $e$ state is $(-1)^{J}$ while the parity of the $f$ state is $(-1)^{J+1}$.

The vibrational quantum numbers used by TROVE (see supplementary material) were correlated to the following standard spectroscopic quantum numbers used for linear-type triatomic molecules: $v_{1}, v_{2}^{\text {lin }}, L=|l|$, $v_{3}$, where $v_{1}$ and $v_{3}$ are the symmetric and asymmetric stretching quantum numbers, respectively, $v_{2}$ is the bending vibrational quantum number used for linear molecules and $l$ is the corresponding vibrational quantum number. The two bending quantum numbers $v_{2}^{\text {lin }}$ and $l$ are connected to the 'non-linear' bending quantum number $v_{2}$ by $v_{2}^{\text {lin }}=2 v_{2}+l$ with $L=v_{2}^{\operatorname{lin}}, v_{2}^{\operatorname{lin}}-2, \ldots 0(1)$.

The symmetries of the vibrational and rotational contributions span the $A_{1}, A_{2}, B_{1}$ and $B_{2}$ irreducible representations (irreps) in $\boldsymbol{C}_{2 \mathrm{v}}(\mathrm{M})$ and $\Sigma_{g / u}^{+/-}(L=0), \Pi_{g / u}(L=1), \Delta_{g / u}(L=2)$ etc. in $\boldsymbol{D}_{\infty \mathrm{h}}$. Odd values of the quantum number $v_{3}$ indicates vibrational states of $B_{2}$ symmetry. The rotational quantum number $k_{a}$ is constrained to the vibrational angular momentum by $k_{a}=l$.

A total of 32951275437 transitions involving 5688942 energy levels up to $J=255$ were computed for the OYT3 line list. The distribution of lines and levels as a function of $J$ is illustrated in Fig. 1. The largest number of transitions in the OYT3 line list occurs between $J=39 \leftrightarrow 40$, while the number of states peaks at $J=46$ before smoothly decreasing, a result of the upper state energy threshold of $h c \cdot 21000 \mathrm{~cm}^{-1}$. 

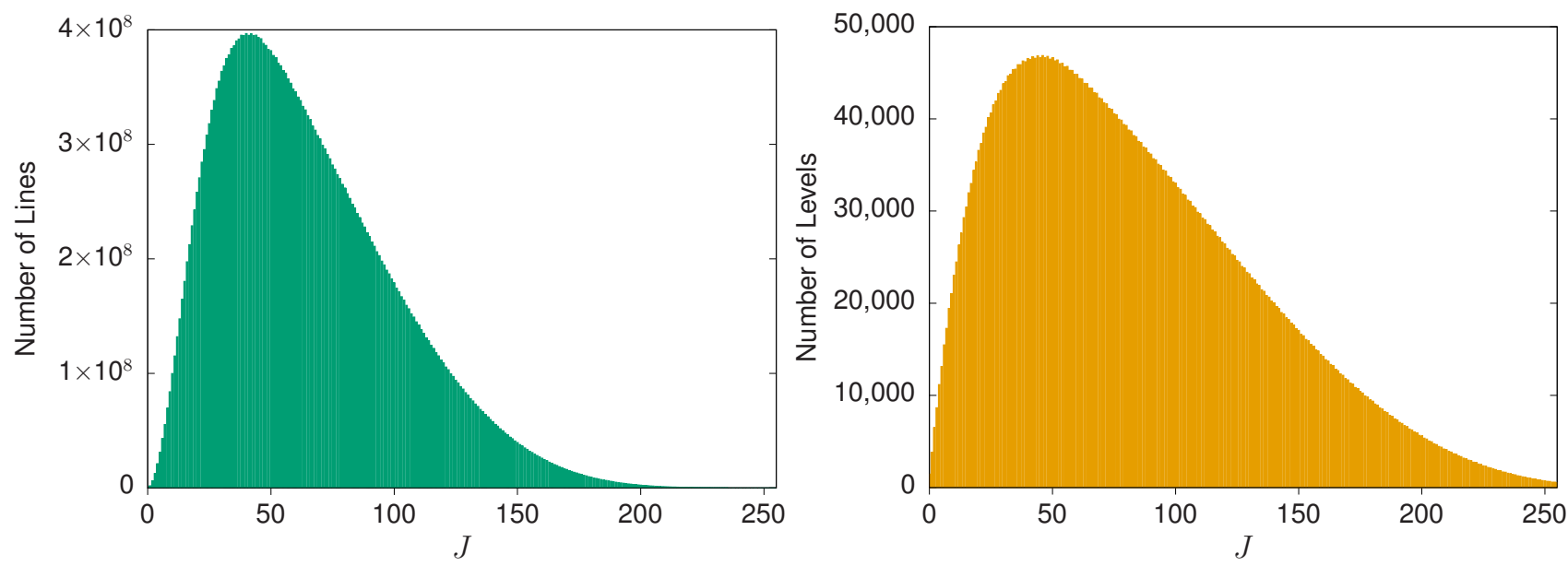

Figure 1. The total number of lines (left panel) and energy levels (right panel) for each value of the rotational quantum number $J$ in the OYT3 line list. For the number of lines, a single $J$ value counts transitions between $J \leftrightarrow J-1$ and $J \leftrightarrow J$.

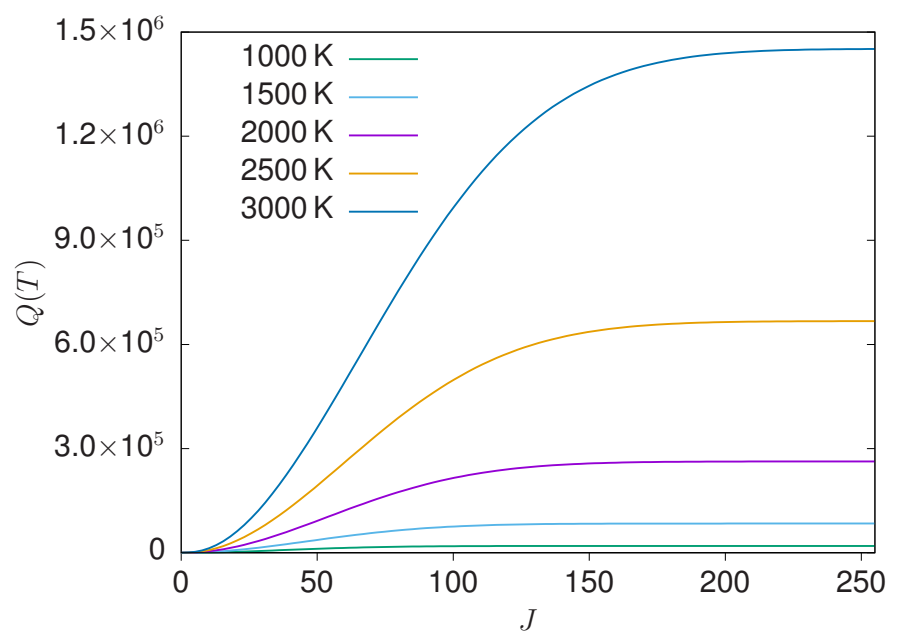

Figure 2. Convergence of the partition function $Q(T)$ of $\mathrm{SiO}_{2}$ with respect to the rotational quantum number $J$ at different temperatures.

\section{RESULTS}

\subsection{Partition function of silicon dioxide}

The temperature-dependent partition function $Q(T)$ is expressed as,

$$
Q(T)=\sum_{i} g_{i} \exp \left(\frac{-E_{i}}{k T}\right),
$$

where $g_{i}=g_{\mathrm{ns}}\left(2 J_{i}+1\right)$ is the degeneracy of a state $i$ with energy $E_{i}$ and rotational quantum number $J_{i}$. Values of the partition function of $\mathrm{SiO}_{2}$ have been computed by summing over all calculated rovibrational energy levels on a $1 \mathrm{~K}$ grid in the $1-3000 \mathrm{~K}$ range (provided as supplementary material). In Fig. 2, the convergence of $Q(T)$ as a function of $J$ for select temperatures is shown. At lower temperatures the partition function converges quickly but a substantial number of high $J$ states must be considered to achieve convergence above $1500 \mathrm{~K}$. At $J=255$, the value of $Q(2000 \mathrm{~K})$ is converged to $0.0001 \%$, while the value of $Q(3000 \mathrm{~K})$ is converged to $0.0019 \%$.

The $\mathrm{SiO}_{2}$ line list was computed with a lower state energy threshold of $h c \cdot 15000 \mathrm{~cm}^{-1}$. A measure of the completeness of the line list can be obtained by studying the reduced partition function $Q_{\text {red }}(T)$, which only includes energy levels up to $h c \cdot 15000 \mathrm{~cm}^{-1}$ in the summation of Eq. (1). The ratio $Q_{\text {red }}(T) / Q(T)$ has been plotted with respect to temperature in Fig. 3 and from this we see that above $1500 \mathrm{~K}$ the ratio starts to decrease from unity. At $3000 \mathrm{~K}$, the ratio $Q$ red $/ Q=0.90$ and this should be considered as a soft temperature limit for the OYT3 line list. Uses above this will result in a progressive loss of opacity but the missing opacity contribution can be estimated from $Q_{\text {red }} / Q$ if needed (Neale, Miller \& Tennyson 1996 ). 


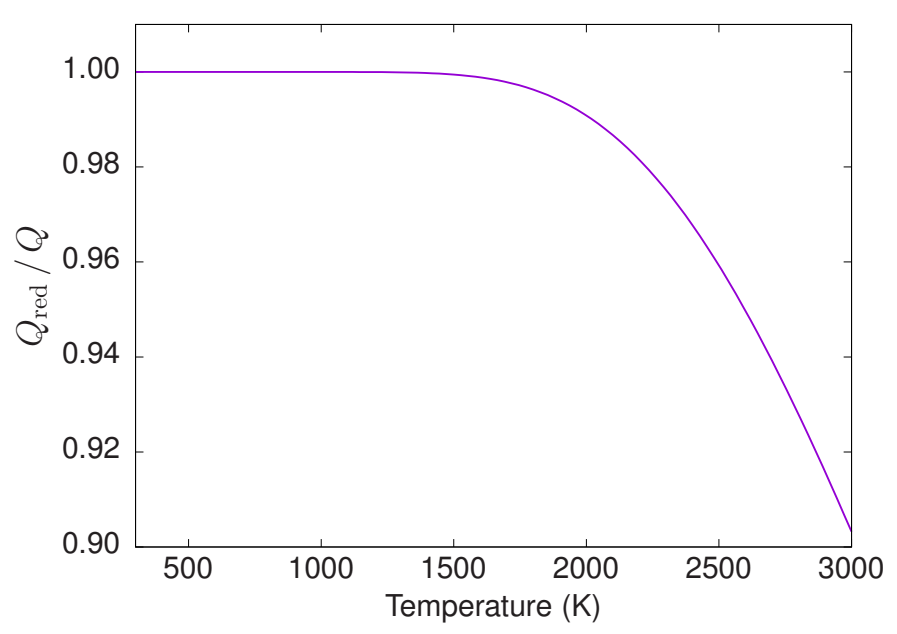

Figure 3. The ratio $Q_{\text {red }} / Q$ as a function of temperature $T$; this provides a measure of the completeness of the OYT3 line list.

Table 1. Extract from the .states file of the $\mathrm{SiO}_{2}$ OYT3 line list.

\begin{tabular}{rrrlllllllllllllllll}
\hline \hline$i$ & $\tilde{E}$ & $g_{\text {tot }}$ & $J$ & unc & $\Gamma_{\text {tot }}$ & $e / f$ & $v_{1}$ & $v_{2}^{\text {lin }}$ & $L$ & $v_{3}$ & $C_{i}$ & $n_{1}$ & $n_{2}$ & $n_{3}$ & $\Gamma_{\text {vib }}$ & $K$ & $\Gamma_{\text {rot }}$ \\
\hline 1 & 0.000000 & 1 & 0 & 0.000000 & $\mathrm{~A} 1$ & $\mathrm{e}$ & 0 & 0 & 0 & 0 & 1.00 & 0 & 0 & 0 & $\mathrm{~A} 1$ & 0 & $\mathrm{~A} 1$ \\
2 & 578.229349 & 1 & 0 & 2.000000 & $\mathrm{~A} 1$ & $\mathrm{e}$ & 0 & 2 & 0 & 0 & 1.00 & 0 & 0 & 1 & $\mathrm{~A} 1$ & 0 & $\mathrm{~A} 1$ \\
3 & 990.856966 & 1 & 0 & 2.000000 & $\mathrm{~A} 1$ & $\mathrm{e}$ & 1 & 0 & 0 & 0 & 1.00 & 1 & 0 & 0 & $\mathrm{~A} 1$ & 0 & $\mathrm{~A} 1$ \\
4 & 1154.529779 & 1 & 0 & 4.000000 & $\mathrm{~A} 1$ & $\mathrm{e}$ & 0 & 4 & 0 & 0 & 1.00 & 0 & 0 & 2 & $\mathrm{~A} 1$ & 0 & $\mathrm{~A} 1$ \\
5 & 1570.947837 & 1 & 0 & 4.000000 & $\mathrm{~A} 1$ & $\mathrm{e}$ & 1 & 2 & 0 & 0 & 1.00 & 1 & 0 & 1 & $\mathrm{~A} 1$ & 0 & $\mathrm{~A} 1$ \\
6 & 1728.958462 & 1 & 0 & 6.000000 & $\mathrm{~A} 1$ & $\mathrm{e}$ & 0 & 6 & 0 & 0 & 1.00 & 0 & 0 & 3 & $\mathrm{~A} 1$ & 0 & $\mathrm{~A} 1$ \\
7 & 1977.509720 & 1 & 0 & 4.000000 & $\mathrm{~A} 1$ & $\mathrm{e}$ & 2 & 0 & 0 & 0 & 1.00 & 1 & 1 & 0 & $\mathrm{~A} 1$ & 0 & $\mathrm{~A} 1$ \\
8 & 2148.937437 & 1 & 0 & 6.000000 & $\mathrm{~A} 1$ & $\mathrm{e}$ & 1 & 4 & 0 & 0 & 1.00 & 1 & 0 & 2 & $\mathrm{~A} 1$ & 0 & $\mathrm{~A} 1$ \\
9 & 2301.592712 & 1 & 0 & 8.000000 & $\mathrm{~A} 1$ & $\mathrm{e}$ & 0 & 8 & 0 & 0 & 1.00 & 0 & 0 & 4 & $\mathrm{~A} 1$ & 0 & $\mathrm{~A} 1$ \\
10 & 2559.419897 & 1 & 0 & 6.000000 & $\mathrm{~A} 1$ & $\mathrm{e}$ & 2 & 2 & 0 & 0 & 1.00 & 1 & 1 & 1 & $\mathrm{~A} 1$ & 0 & $\mathrm{~A} 1$ \\
\hline \hline
\end{tabular}

$i$ : State counting number;

$\tilde{E}$ : Term value $\left(\right.$ in $\mathrm{cm}^{-1}$ );

$g_{\text {tot }}:$ Total state degeneracy;

$J$ : Total angular momentum quantum number;

unc: Estimated uncertainty of energy level $\left(\right.$ in $\left.\mathrm{cm}^{-1}\right)$;

$\Gamma_{\text {tot }}$ : Overall symmetry in $C_{2 \mathrm{v}}(\mathrm{M})\left(A_{1}\right.$ or $\left.A_{2}\right)$;

$e / f$ : The Kronig (rotationless) parity;

$v_{1}, v_{2}^{\text {lin }}, L, v_{3}$ : Linear-molecule vibrational quantum numbers;

$C_{i}$ : Largest coefficient used in the TROVE assignment;

$n_{1}-n_{3}$ : TROVE vibrational quantum numbers;

$\Gamma_{\text {vib }}$ : Symmetry of the vibrational contribution in $\boldsymbol{C}_{2 \mathrm{v}}(\mathrm{M})$;

$K$ : Rotational quantum number, projection of $J$ onto molecule-fixed $z$ axis $(K=L)$;

$\Gamma_{\text {rot }}$ : Symmetry of the rotational contribution in $\boldsymbol{C}_{2 \mathrm{v}}(\mathrm{M})$.

\subsection{Line list format}

The $\mathrm{SiO}_{2}$ line list is provided in the ExoMol data format and further details with illustrative examples can be found in Tennyson et al. (2016). The .states file, see Table 1, contains all the computed rovibrational energy levels $\left(\right.$ in $\mathrm{cm}^{-1}$ ), each labelled with a unique state ID counting number, symmetry and quantum number labelling, and the contribution $C_{i}$ from the largest eigencoefficient used to assign the rovibrational state. The .trans files have been split into $100 \mathrm{~cm}^{-1}$ frequency bins for user-handling purposes and contain all computed transitions with upper and lower state ID labels and Einstein $A$ coefficients, see Table 2 .

The assignment of the vibrational quantum numbers to each state is performed in TROVE by analysing the contribution from the primitive basis functions of the different modes, which are then converted to the linear-molecule, normal mode quantum numbers (see Table 1). The connection between the assignment and the primitive basis functions is not always straightforward due to the complicated contraction scheme used to build the final symmetrized rovibrational basis set (Yurchenko, Yachmenev \& Ovsyannikov 2017). Thus, in instances where the eigen-coefficient $\left|C_{i}\right|$ is very small the assignment should be considered as indicative.

The computed energy levels in the OYT3 line list have been assigned uncertainties (in $\mathrm{cm}^{-1}$ ) in the following way: The three fundamental term values have been given an estimated uncertainty of $2 \mathrm{~cm}^{-1}$, which has then been propagated to all 
Table 2. Extract from the .trans file for the $0-100 \mathrm{~cm}^{-1}$ window of the $\mathrm{SiO}_{2}$ OYT3 line list.

\begin{tabular}{rrc}
\hline \hline \multicolumn{1}{c}{$f$} & \multicolumn{1}{c}{$i$} & $A_{i f}$ \\
\hline 1901572 & 1832882 & $8.4692 \mathrm{e}-44$ \\
2283835 & 2261046 & $9.7538 \mathrm{e}-45$ \\
948596 & 1016760 & $9.4147 \mathrm{e}-35$ \\
1830303 & 1853859 & $2.7761 \mathrm{e}-42$ \\
4120223 & 4135284 & $1.1104 \mathrm{e}-41$ \\
649389 & 670531 & $1.0237 \mathrm{e}-43$ \\
284492 & 332334 & $2.6832 \mathrm{e}-31$ \\
3288298 & 3306461 & $1.3021 \mathrm{e}-31$ \\
3796784 & 3812806 & $2.0901 \mathrm{e}-39$ \\
366209 & 348742 & $3.7795 \mathrm{e}-33$ \\
\hline \hline
\end{tabular}

$f$ : Upper state ID; $i$ : Lower state ID;

$A_{i f}$ : Einstein $A$ coefficient (in $\mathrm{s}^{-1}$ ).

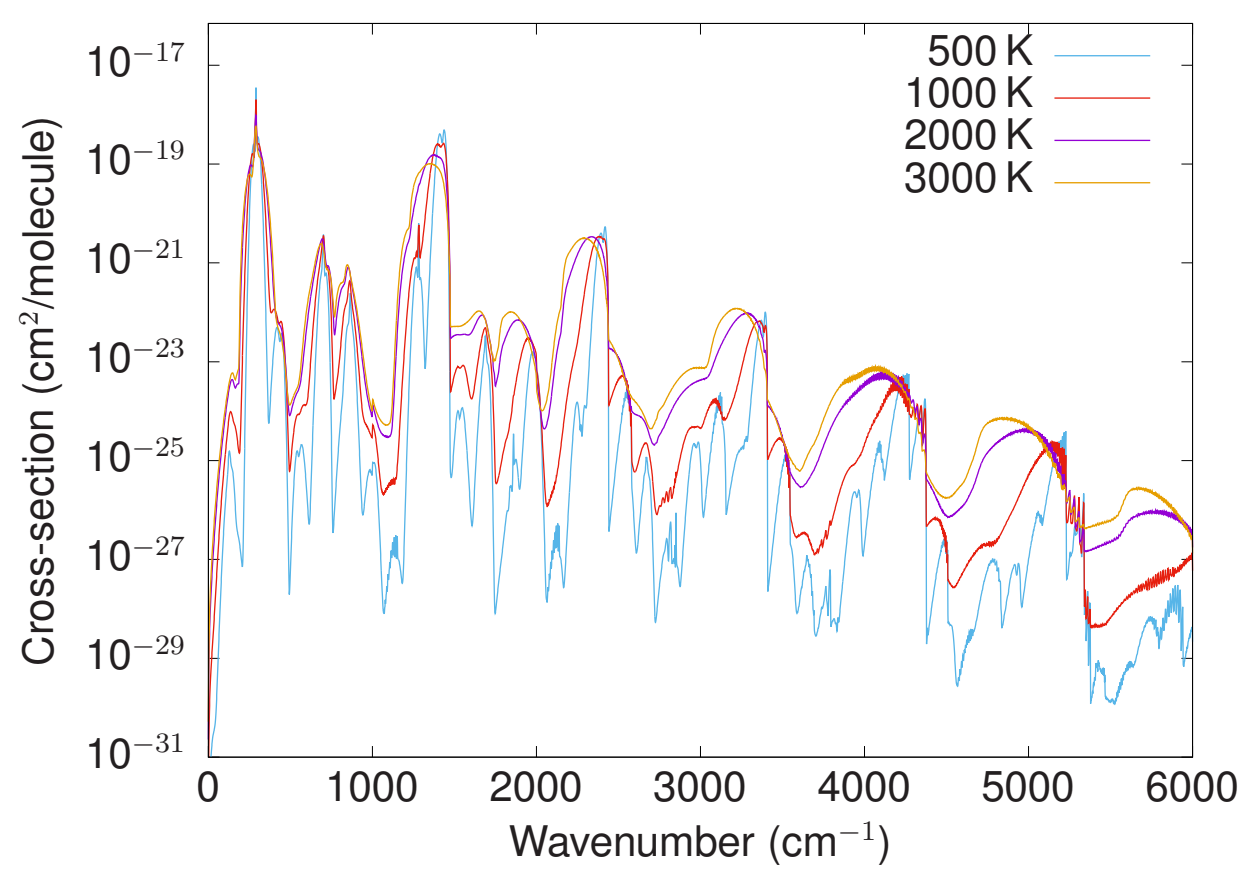

Figure 4. Temperature dependence of the spectrum of $\mathrm{SiO}_{2}$, which becomes increasingly flat as the temperature increases. Absorption cross-sections were computed from the OYT3 line list and represented with a Gaussian line profile with a half width at half maximum (HWHM) of $1 \mathrm{~cm}^{-1}$ at a resolution of $1 \mathrm{~cm}^{-1}$.

overtone and combination bands using the TROVE normal mode quantum numbers. For example, a state with $\left(n_{1}=5\right.$, $n_{2}=2, n_{3}=1$ ) has an estimated uncertainty of $16 \mathrm{~cm}^{-1}$. The initial uncertainty estimate of $2 \mathrm{~cm}^{-1}$ for the fundamentals is based on our previous experience using similar levels of ab initio theory to construct closed-shell molecule potential energy surfaces (Owens et al. 2015a,b, 2016, 2018; Owens \& Yurchenko 2019). This uncertainty scheme is approximate and should not be relied on in instances where the eigen-coefficient $\left|C_{i}\right|$ is small. We have also opted to round the estimated uncertainties to integer values so that they can be easily differentiated from more robust uncertainties, e.g. derived from experiment. Note that the ground state $J>0$ rovibrational term values, i.e. $\left(n_{1}=0, n_{2}=0, n_{3}=0\right)$ have been assigned uncertainties of $2 \mathrm{~cm}^{-1}$ throughout.

\subsection{Simulated spectra of silicon dioxide}

The temperature dependence of the OYT3 line list is illustrated in Fig. 4, where we have plotted integrated absorption crosssections at a resolution of $1 \mathrm{~cm}^{-1}$ using a Gaussian profile with a half width at half maximum (HWHM) of $1 \mathrm{~cm}^{-1}$. Spectral simulations were performed with the ExoCross program (Yurchenko, Al-Refaie \& Tennyson 2018). As expected, the $\mathrm{SiO}_{2}$ spectrum becomes smoother and more featureless as the temperature increases. This is caused by the increased population of vibrationally excited states with temperature, leading to substantial broadening of the rotational band envelopes.

Stick spectra of the two strongest bands at a temperature of $1000 \mathrm{~K}$ are shown in Fig. 5, with the fundamental $\nu_{2}$ 

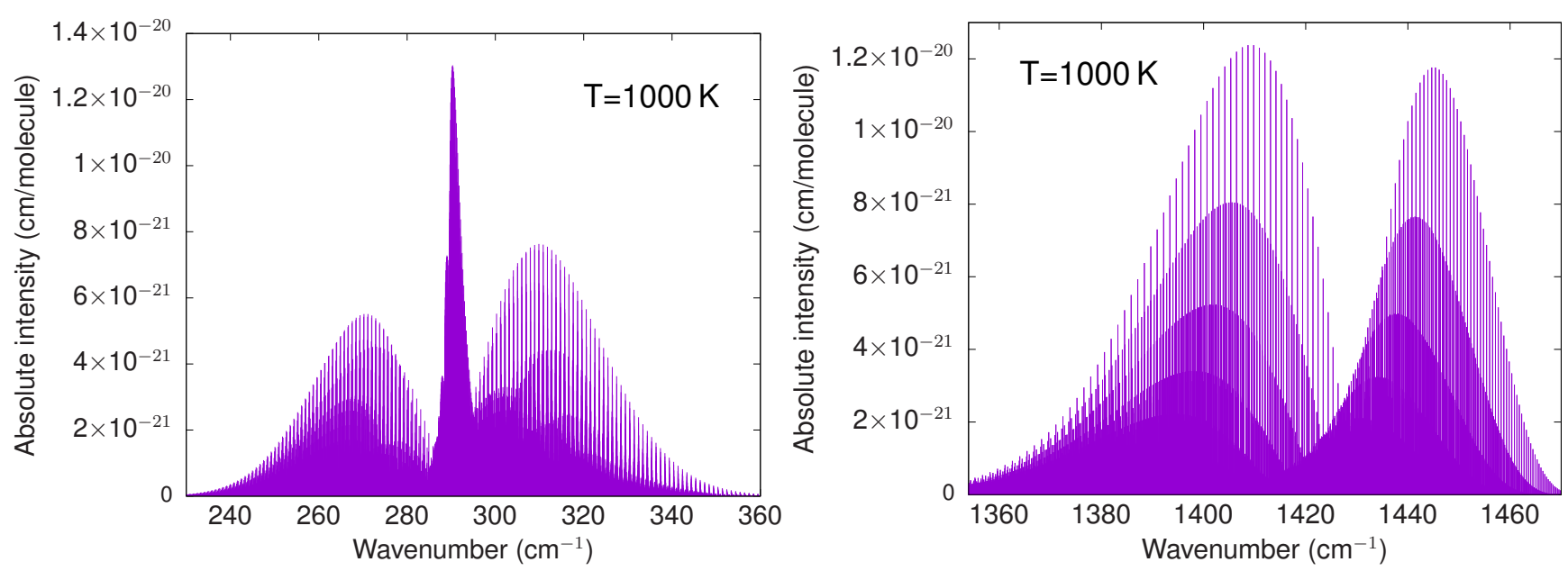

Figure 5. Stick spectrum of the two strongest bands of $\mathrm{SiO}_{2}$ at $T=1000 \mathrm{~K}$. The left panel shows the bending $\nu_{2}(0,1,1,0)$ fundamental band while the right panel shows the stretching $\nu_{3}(0,0,0,1)$ band, where states are labelled by the quantum numbers $\left(v_{1}, v_{2}, L, v_{3}\right)$.

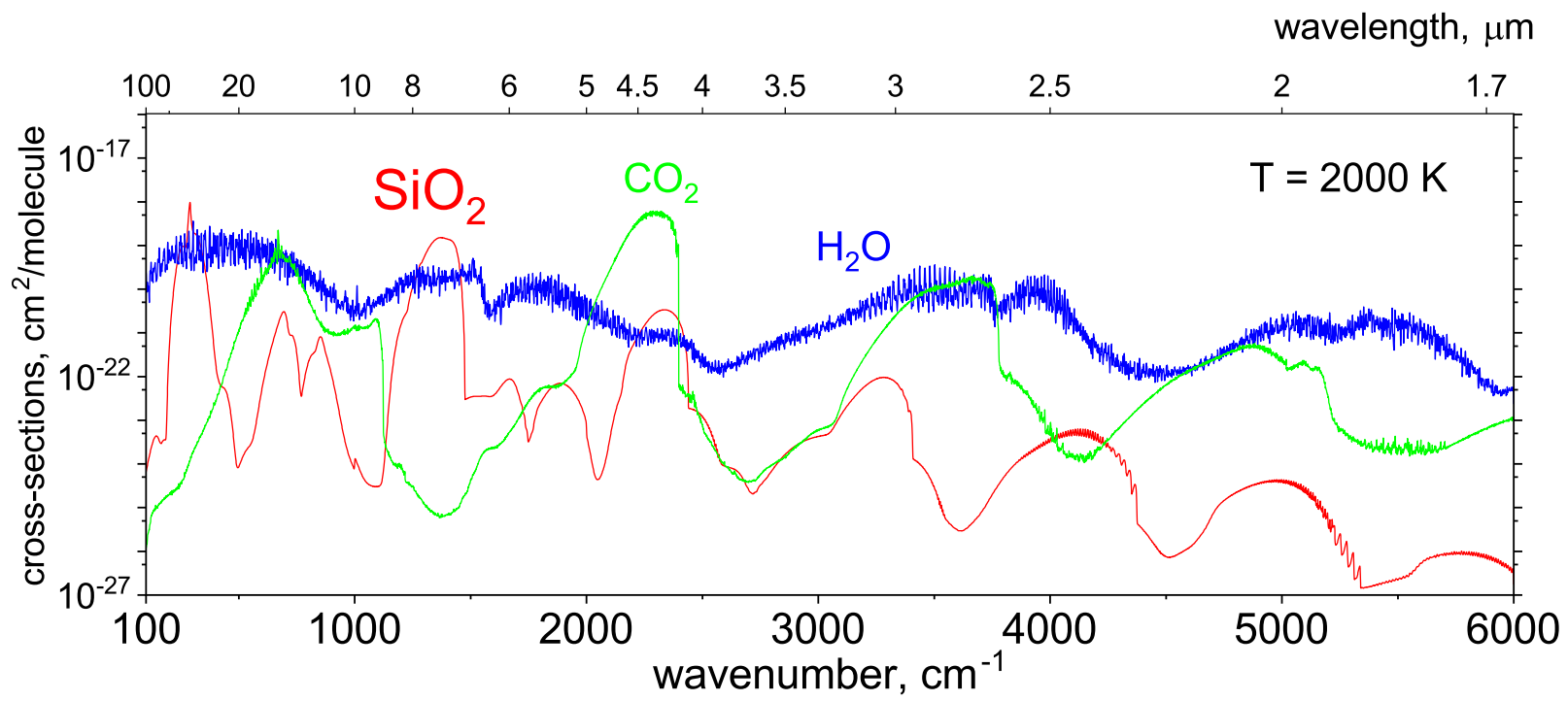

Figure 6. Comparison of the $2000 \mathrm{~K}$ broadband spectra of $\mathrm{SiO}_{2}, \mathrm{CO}_{2}$ and water. A Gaussian line profile with $\mathrm{HWHM}$ of $1 \mathrm{~cm}^{-1}$ at a resolution of $1 \mathrm{~cm}^{-1}$ was used. The $\mathrm{CO}_{2}$ cross sections were computed using the new UCL-4000 line list (Yurchenko et al. 2020) and Pokazatel line list for water (Polyansky et al. 2018). For clarity the relatively flat water spectrum has been reduced by a factor of ten. $\mathrm{SiO}_{2}$ shows strong absorption features around 250 and $1500 \mathrm{~cm}^{-1}$. A weaker $\mathrm{SiO}_{2}$ feature at about $2300 \mathrm{~cm}^{-1}$ is masked by strong $\mathrm{CO}_{2}$ absorption in this region.

bending mode (left-hand panel) and the stretching $\nu_{3}$ band (right-hand panel) displayed. The position of these bands is in broad agreement with previous experimental studies (Andrews \& McCluskey 1992; Schnöckel 1978; Schnöckel 1980) but given these infrared measurements were performed in solid argon matrices, which are known to shift the measured wavenumbers, we avoid a direct comparison.

The $\mathrm{SiO}_{2}$ spectrum has a distinct strong feature at $4.5 \mu \mathrm{m}$. This region plays an important role in the atmospheric applications of exoplanets due to the $\mathrm{CO}_{2}$ photometric band used by the Spitzer Space Telescope (IRAC instrument). For example, this band was used to build the phase curve of super-Earth 55 Cancri e by Demory et al. (2016) and to provide analysis of the atmospheric (day/night) structure of the plane. The atmosphere was shown to have a high temperature contrast, from $1400 \mathrm{~K}$ (night side) to $2700 \mathrm{~K}$ (day side). In Fig. 6, we show that the $\mathrm{CO}_{2} 4.5 \mu \mathrm{m}$ region has strong overlap with the spectrum of $\mathrm{SiO}_{2}$, namely the $\left(1,4^{0}, 0\right)$ band in the same region, although the strongest absorption bands are $\left(0,1^{1}, 0\right)$ and $\left(0,2^{2}, 0\right)$ where states are labelled by the quantum numbers $\left(v_{1}, v_{2}^{L}, v_{3}\right)$. An absorption spectrum of water is also shown for comparison. Silicon dioxide has also been suggested as a potential constituent of the atmosphere of the super-Earth Corot-7b (Schaefer, Lodders \& Fegley 2012) and thus should be included in retrievals for hot super-Earths. 


\section{CONCLUSION}

A comprehensive molecular line list for $\mathrm{SiO}_{2}$ has been presented. The line list, named OYT3, covers the $0-6000 \mathrm{~cm}^{-1}$ range (wavelengths $\lambda>1.67 \mu \mathrm{m}$ ) for states below $J=255$ and is applicable for temperatures up to $3000 \mathrm{~K}$. As discussed above, the lack of reliable experimental spectroscopic data on $\mathrm{SiO}_{2}$ has meant that the OYT3 line list has been constructed using purely $a b$ initio methods with no degree of empirical refinement. The accuracy of the predicted line positions will suffer as a result, particularly for highly excited states and shorter wavelengths, but for the fundamental bands, which have the strongest intensity, the errors should be within $1-3 \mathrm{~cm}^{-1}$ as a conservative estimate. The computed line intensities should not be overly affected and are largely expected to be within the $5-10 \%$ of experimentally determined intensities. Of course, without reliable experimental data to compare against these are only estimates based on our previous experience constructing ab initio spectroscopic models with similar electronic structure methods (see, for example, our work on $\mathrm{SiH}_{4}\left(\mathrm{Owens}_{\mathrm{s}}\right.$ et al. 2015b).

The usual ExoMol methodology is to take advantage of laboratory measurements to improve the accuracy of our computed line lists (Tennyson 2012). However, in the absence of high-resolution spectroscopic measurements for $\mathrm{SiO}_{2}$, this has not proved possible. In practice a number of current line lists are entirely ab initio. Of course, for few electron systems such as $\mathrm{HD}^{+}$ (Amaral et al. 2019), HD (Amaral et al. 2019), $\mathrm{HeH}^{+}$(Engel et al. 2005; Amaral et al. 2019), H3 (Mizus et al. 2017) and $\mathrm{LiH}$ (Coppola, Lodi \& Tennyson 2011) it is possible to compute high accuracy line lists which should reproduce astronomical spectra within their observational accuracy. More pertinent here is the case of HCN. The original HCN / HNC line list of Harris, Polyansky \& Tennyson (2002) was based on a purely ab initio potential energy and dipole surfaces (van Mourik et al. 2001) computed at a lower level of theory, and hence of lower accuracy, than that employed here. This line list has been successively updated (Harris et al. 2006; Barber et al. 2014), and even adapted to $\mathrm{H}^{13} \mathrm{CN}$ (Harris et al. 2008), by the post hoc insertion of empirical energy levels, something that is explicitly allowed for in the ExoMol data format (Tennyson, Hill \& Yurchenko 2013). The HCN line list both in its original and updated forms has proved to be highly useful and indeed underpins a number of recent (possible) detections of HCN in exoplanets (Tsiaras et al. 2016; Hawker et al. 2018; Gandhi et al. 2020) and much exoplanet modelling; it has also been found useful for combustion studies (Glarborg \& Marshall 2017). We would anticipate the OYT3 $\mathrm{SiO}_{2}$ line list being used in a similar fashion and, should high resolution $\mathrm{SiO}_{2}$ spectra become available, we will update the line list to improve its accuracy. However, we are unaware of any such studies in progress at present.

For present use, we recommend the OYT3 line list for low-resolution studies of exoplanet atmospheres, e.g. with a resolving power of $R \approx 100$, but we emphasise that the line list is not designed for high-resolution analysis. Interestingly, a recent cold molecular beam study has shown that silicon dioxide can be efficiently formed through the reaction of $\mathrm{SiH}_{\text {and }} \mathrm{O}_{2}$ under single collision conditions (Yang et al. 2018), demonstrating a low-temperature pathway to gas-phase $\mathrm{SiO}_{2}$ that is plausible in the interstellar medium or molecular clouds. The OYT3 line list will aid other astronomical searches for $\mathrm{SiO}_{2}$ and may find use in industrial processes, for example, in the semiconductor industry where silicon-bearing molecules are commonly encountered.

\section{ACKNOWLEDGMENTS}

This work was supported by the STFC Projects No. ST/M001334/1 and ST/R000476/1. The authors acknowledge the use of the UCL Legion High Performance Computing Facility (Legion@UCL) and associated support services in the completion of this work, along with the Cambridge Service for Data Driven Discovery (CSD3), part of which is operated by the University of Cambridge Research Computing on behalf of the STFC DiRAC HPC Facility (www.dirac.ac.uk). The DiRAC component of CSD3 was funded by BEIS capital funding via STFC capital grants ST/P002307/1 and ST/R002452/1 and STFC operations grant ST/R00689X/1. DiRAC is part of the National e-Infrastructure.

\section{REFERENCES}

Adler T. B., Knizia G., Werner H. J., 2007, J. Chem. Phys., 127, 221106

Amaral P. H. R., Diniz L. G., Jones K. A., Stanke M., Alijah A., Adamowicz L., Mohallem J. R., 2019, ApJ, 878, 95

Andrews L., McCluskey M., 1992, J. Mol. Spectrosc., 154, 223

Barber R. J., Strange J. K., Hill C., Polyansky O. L., Mellau G. C., Yurchenko S. N., Tennyson J., 2014, MNRAS, 437, 1828

Barton E. J., Yurchenko S. N., Tennyson J., 2013, MNRAS, 434, 1469

Brinkmann N. R., Tschumper G. S., Schaefer III H. F., 1999, J. Chem. Phys., 110, 6240

Brown J. M. et al., 1975, J. Mol. Spectrosc., 55, 500

Bunker P. R., Jensen P., 1998, Molecular Symmetry and Spectroscopy, 2nd edn. NRC Research Press, Ottawa

Carter S., Handy N., Sutcliffe B., 1983, Mol. Phys., 49, 745

CFOUR, 2019. A quantum chemical program package written by J. F. Stanton, J. Gauss, M. E. Harding, and P. G. Szalay with contributions from A. A. Auer, R. J. Bartlett, U. Benedikt, C. Berger, D. E. Bernholdt, Y. J. Bomble, L. Cheng, O. Christiansen, M. Heckert, O. Heun, C. Huber, T.-C. Jagau, D. Jonsson, J. Jusélius, K. Klein, W. J. Lauderdale, D. A. Matthews, T. Metzroth, L. 
A. Mück, D. P. O'Neill, D. R. Price, E. Prochnow, C. Puzzarini, K. Ruud, F. Schiffmann, W. Schwalbach, S. Stopkowicz, A. Tajti, J. Vázquez, F. Wang, J. D. Watts, and the integral packages MOLECULE (J. Almlöf and P. R. Taylor), PROPS (P. R. Taylor), ABACUS (T. Helgaker, H. J. Aa. Jensen, P. Jørgensen, and J. Olsen), and ECP routines by A. V. Mitin and C. van Wüllen. For the current version, see http://www.cfour.de.

Chubb K. L., Yachmenev A., Tennyson J., Yurchenko S. N., 2018, J. Chem. Phys., 149, 014101

Clark V. H. J., Owens A., Tennyson J., Yurchenko S. N., 2020, J. Quant. Spectrosc. Radiat. Transf., 246, 106929

Cooley J. W., 1961, Math. Comp., 15, 363

Coppola C. M., Lodi L., Tennyson J., 2011, MNRAS, 415, 487

Császár A. G., Allen W. D., Schaefer III H. F., 1998, J. Chem. Phys., 108, 9751

de Jong W. A., Harrison R. J., Dixon D. A., 2001, J. Chem. Phys., 114, 48

Demory B.-O. et al., 2016, Nature, 532, 207

Douglas M., Kroll N. M., 1974, Ann. Phys., 82, 89

Dunning T. H., 1989, J. Chem. Phys., 90, 1007

Engel E. A., Doss N., Harris G. J., Tennyson J., 2005, MNRAS, 357, 471

Fegley, Jr. B., Jacobson N. S., Williams K. B., Plane J. M. C., Schaefer L., Lodders K., 2016, ApJ, 824, 103

Gandhi S. et al., 2020, MNRAS, 495, 224-237

Glarborg P., Marshall P., 2017, Energy \& Fuels, 31, 2156

Gorman M., Yurchenko S. N., Tennyson J., 2019, MNRAS, 490, 1652

Hao Y., Xie Y., Schaefer III H. F., 2014, RSC Adv., 4, 47163

Harris G. J., Larner F. C., Tennyson J., Kaminsky B. M., Pavlenko Y. V., Jones H. R. A., 2008, MNRAS, 390, 143

Harris G. J., Polyansky O. L., Tennyson J., 2002, ApJ, 578, 657

Harris G. J., Tennyson J., Kaminsky B. M., Pavlenko Y. V., Jones H. R. A., 2006, MNRAS, 367, 400

Hättig C., 2005, Phys. Chem. Chem. Phys., 7, 59

Hawker G. A., Madhusudhan N., Cabot S. H. C., Gandhi S., 2018, ApJL, 863, L11

Heß B. A., 1986, Phys. Rev. A, 33, 3742

Hill J. G., Mazumder S., Peterson K. A., 2010, J. Chem. Phys., 132, 054108

Hill J. G., Peterson K. A., Knizia G., Werner H.-J., 2009, J. Chem. Phys., 131, 194105

Jacox M. E., 1994, Chem. Phys., 189, 149

Jørgensen U. G., Jensen P., 1993, J. Mol. Spectrosc., 161, 219

Kállay M., Gauss J., 2005, J. Chem. Phys., 123, 214105

Kállay M., Gauss J., 2008, J. Chem. Phys., 129, 144101

Kállay M. et al., 2020, J. Chem. Phys., 152, 074107

Kaufman M., Muenter J., Klemperer W., 1967, J. Chem. Phys., 47, 3365

Kostko O., Ahmed M., Metz R. B., 2009, J. Phys. Chem. A, 113, 1225

Miguel Y., Kaltenegger L., Fegley B., Schaefer L., 2011, ApJ, 742, L19

Mizus I. I., Alijah A., Zobov N. F., Kyuberis A. A., Yurchenko S. N., Tennyson J., Polyansky O. L., 2017, MNRAS, 468, 1717

Neale L., Miller S., Tennyson J., 1996, ApJ, 464, 516

Noumerov B. V., 1924, MNRAS, 84, 592

Owens A., Yachmenev A., Küpper J., Yurchenko S. N., Thiel W., 2018, Phys. Chem. Chem. Phys., 21, 3496

Owens A., Yurchenko S. N., 2019, J. Chem. Phys., 150, 194308

Owens A., Yurchenko S. N., Yachmenev A., Tennyson J., Thiel W., 2015a, J. Chem. Phys., 142, 244306

Owens A., Yurchenko S. N., Yachmenev A., Tennyson J., Thiel W., 2016, J. Chem. Phys., 145, 104305

Owens A., Yurchenko S. N., Yachmenev A., Thiel W., 2015b, J. Chem. Phys., 143, 244317

Owens A., Yurchenko S. N., Yachmenev A., Thiel W., Tennyson J., 2017, MNRAS, 471, 5025

Pacansky J., Hermann K., 1978, J. Chem. Phys., 69, 963

Partridge H., Schwenke D. W., 1997, J. Chem. Phys., 106, 4618

Peterson K. A., Adler T. B., Werner H.-J., 2008, J. Chem. Phys., 128, 084102

Polyansky O. L., Kyuberis A. A., Zobov N. F., Tennyson J., Yurchenko S. N., Lodi L., 2018, MNRAS, 480, 2597

Schaefer L., Fegley B., 2009, ApJ, 703, L113

Schaefer L., Lodders K., Fegley, Jr. B., 2012, ApJ, 755, 41

Schnöckel H., 1978, Angew. Chem. Int. Ed. Engl., 17, 616

Schnöckel H., 1980, Z. anorg. allg. Chem., 460, 37

Sutcliffe B. T., Tennyson J., 1991, Intern. J. Quantum Chem., 39, 183

Ten-No S., 2004, Chem. Phys. Lett., 398, 56

Tennyson J., 2012, WIREs Comput. Mol. Sci., 2, 698

Tennyson J., 2014, J. Mol. Spectrosc., 298, 1

Tennyson J., 2016, J. Chem. Phys., 145, 120901

Tennyson J., Hill C., Yurchenko S. N., 2013, in AIP Conference Proceedings, Vol. 1545, $6^{\text {th }}$ international conference on atomic and molecular data and their applications ICAMDATA-2012, AIP, New York, pp. 186-195

Tennyson J., Kostin M. A., Barletta P., Harris G. J., Polyansky O. L., Ramanlal J., Zobov N. F., 2004, Comput. Phys. Commun., 163, 85

Tennyson J., Sutcliffe B. T., 1982, J. Chem. Phys., 77, 4061

Tennyson J., Sutcliffe B. T., 1986, Mol. Phys., 58, 1067

Tennyson J., Sutcliffe B. T., 1992, Intern. J. Quantum Chem., 42, 941

Tennyson J., Yurchenko S. N., 2012, MNRAS, 425, 21

Tennyson J., Yurchenko S. N., 2017a, Mol. Astrophys., 8, 1

Tennyson J., Yurchenko S. N., 2017b, Intern. J. Quantum Chem., 117, 92

Tennyson J., Yurchenko S. N., 2018, Atoms, 6, 26 
Tennyson J. et al., 2016, J. Mol. Spectrosc., 327, 73

Tsiaras A. et al., 2016, ApJ, 820, 99

Tyuterev V. G., Tashkun S. A., Schwenke D. W., 2001, Chem. Phys. Lett., 348, 223

Upadhyay A., Conway E. K., Tennyson J., Yurchenko S. N., 2018, MNRAS, 477, 1520

van Mourik T., Harris G. J., Polyansky O. L., Tennyson J., Császár A. G., Knowles P. J., 2001, J. Chem. Phys., 115, 3706

Wang L.-S., Wu H., Desai S. R., Fan J., Colson S. D., 1996, J. Phys. Chem., 100, 8697

Watson J. K. G., 2003, J. Mol. Spectrosc., 219, 326

Weigend F., 2002, Phys. Chem. Chem. Phys., 4, 4285

Werner H.-J., Knowles P. J., Knizia G., Manby F. R., Schütz M., 2012, WIREs Comput. Mol. Sci., 2, 242

Yachmenev A., Yurchenko S. N., 2015, J. Chem. Phys., 143, 014105

Yachmenev A., Yurchenko S. N., Ribeyre T., Thiel W., 2011, J. Chem. Phys., 135, 074302

Yang T., Thomas A. M., Dangi B. B., Kaiser R. I., Mebel A. M., Millar T. J., 2018, Nat. Commun., 9

Yousaf K. E., Peterson K. A., 2008, J. Chem. Phys., 129, 184108

Yurchenko S. N., 2014, in Chemical Modelling: Volume 10, Vol. 10, The Royal Society of Chemistry, pp. 183-228

Yurchenko S. N., Al-Refaie A. F., Tennyson J., 2018, A\&A, 614, A131

Yurchenko S. N., Barber R. J., Yachmenev A., Thiel W., Jensen P., Tennyson J., 2009, J. Phys. Chem. A, 113, 11845

Yurchenko S. N., Tennyson J., Miller S., Melnikov V. V., O’Donoghue J., Moore L., 2020, MNRAS

Yurchenko S. N., Thiel W., Jensen P., 2007, J. Mol. Spectrosc., 245, 126

Yurchenko S. N., Yachmenev A., Ovsyannikov R. I., 2017, J. Chem. Theory Comput., 13, 4368

\section{SUPPORTING INFORMATION}

Supplementary data are available at MNRAS online. This includes the potential energy and dipole moment surfaces of $\mathrm{SiO}_{2}$ with programs to construct them, and values of the temperature-dependent partition function of $\mathrm{SiO}_{2}$ up to $3000 \mathrm{~K}$. The following references were cited in the supplementary material: (Owens et al. 2015a,b, 2016, 2018; Owens \& Yurchenko 2019; Császár, Allen \& Schaefer III 1998; Adler, Knizia \& Werner 2007; Peterson, Adler \& Werner 2008; Hill et al. 2009; Ten-No 2004; Yousaf \& Peterson 2008; Weigend 2002; Hättig 2005; Werner et al. 2012; Yachmenev et al. 2011; Hill, Mazumder \& Peterson 2010; Kállay \& Gauss 2005, 2008; Kállay et al. 2020; CFOUR 2019; Dunning 1989; Douglas \& Kroll 1974; Heß 1986; de Jong, Harrison \& Dixon 2001; Tyuterev, Tashkun \& Schwenke 2001; Partridge \& Schwenke 1997; Watson 2003; Jørgensen \& Jensen 1993; Yurchenko, Thiel \& Jensen 2007; Yurchenko et al. 2009; Yachmenev \& Yurchenko 2015; Tennyson \& Yurchenko 2017b; Yurchenko, Yachmenev \& Ovsyannikov 2017; Chubb et al. 2018; Carter, Handy \& Sutcliffe 1983; Sutcliffe \& Tennyson 1991; Noumerov 1924; Cooley 1961; Tennyson \& Sutcliffe 1986; Tennyson et al. 2004; Tennyson \& Sutcliffe 1992, 1982; Yurchenko, Al-Refaie \& Tennyson 2018) 